\title{
Can finite-frequency effects be accounted for in ray theory surface wave tomography?
}

\author{
Anne Sieminski, Jean-Jacques Lévêque, and Eric Debayle \\ Ecole et Observatoire des Sciences de la Terre, CNRS and Université Louis Pasteur, Strasbourg, France \\ Received 2 September 2004; revised 27 October 2004; accepted 29 November 2004; published 29 December 2004.
}

[1] We present a series of synthetic tests showing that regional surface wave tomographies with a dense path coverage of the target region can be safely conducted under ray theory because the shortcomings of ray theory in considering finite-frequency effects can be counterbalanced by a physically-based regularization of the inversion. In particular, we show that with ray theory applied under the above conditions, it is possible to detect heterogeneities with length scales smaller than the wavelength of the data set. INDEX TERMS: 7255 Seismology: Surface waves and free oscillations; 7260 Seismology: Theory and modeling; 8180 Tectonophysics: Tomography. Citation: Sieminski, A., J.-J. Lévêque, and E. Debayle (2004), Can finite-frequency effects be accounted for in ray theory surface wave tomography?, Geophys. Res. Lett., 31, L24614, doi:10.1029/2004GL021402.

\section{Introduction}

[2] Most of the present day surface wave tomographies are based on the assumption that surface waves propagate along the source-station great circle without mode-coupling and that they are only sensitive to the structure along the zero-width ray [e.g., Trampert and Woodhouse, 1995; Ritsema et al., 2004; Debayle and Sambridge, 2004]. This "great circle ray theory" has been widely used because it provides a simple theoretical framework for the efficient analysis of large volumes of data. The obvious drawback of retrieving information only along the ray paths is generally overcome by extending the properties found along the rays to their immediate vicinity. This can be done through the parameterization of the model, for example by spreading to a whole block what is found for a ray crossing the block, and/or by adding some kind of regularization into the inverse operator. In this context, retrieving small scale details, such as heterogeneities smaller than a wavelength, is in principle precluded because of the poor description of the surface wave sensitivity, and also because ray theory is valid only for a medium that does not vary significantly over a wavelength (or the Fresnel zone width) [e.g., Spetzler et al., 2002]. More accurate descriptions of wave propagation have been proposed in an attempt to obtain higher resolution images. For surface wave tomography, the focus has been on taking into account a finite-size sensitivity zone around the paths [Spetzler et al., 2002; Ritzwoller et al., 2002; Yoshizawa and Kennett, 2002].

[3] This paper addresses whether the theoretical shortcomings of ray theory in considering finite-frequency effects can be counterbalanced by other factors, such as a dense ray coverage or a physically-based choice of regularization scheme. We explore, using a series of surface wave tomography synthetic tests, whether inverting the data set using a finite-frequency theory provides a significant improvement compared to great circle ray theory combined with a physically-based regularization. We use a representation of the finite-frequency effects based on single scattering theory.

\section{Finite-Size Sensitivity Zones}

[4] In great circle ray theory the phase slowness $1 / c^{\text {obs }}$ observed for each path is interpreted as the great circle pathaverage slowness

$$
\frac{1}{c^{o b s}}=\frac{1}{\Delta_{0}} \int_{\Delta_{0}} \frac{1}{c(s)} d s
$$

with $\Delta_{0}$ the distance along the great circle and $1 / c(s)$ the local phase slowness.

[5] To account for finite-frequency effects the observed phase slowness has to be interpreted as a weighted average over a $2 \mathrm{D}$ zone

$$
\frac{1}{c^{o b s}}=\int_{\Delta_{0}} \int_{L(s)} K(s, n) \frac{1}{c(s, n)} d n d s
$$

where the weighting function (sensitivity kernel) $K(s, n)$ describes the sensitivity to the structure and $L(s)$ is the width of the 2D zone. Different definitions of $K(s, n)$ and $L(s)$ have been proposed [Spetzler et al., 2002; Yoshizawa and Kennett, 2002; Ritzwoller et al., 2002]. We follow here a description similar to that of Spetzler et al. [2002], based on the linearized scattering theory developed by Snieder [2002], which only considers near forward, single scattering (first Born-Rytov approximation) without mode coupling nor source directivity effect.

[6] In classical surface wave analysis, $c^{o b s}$ is measured via a gaussian band-pass filtering of the seismograms [e.g., Dziewonski et al., 1969], and is actually a gaussian average over a frequency-band. The corresponding sensitivity kernel should therefore be the integral of the scattering kernel weighted in frequency by the same filter, as suggested by Spetzler et al. [2002]. A side effect of the summation happens to be a significant attenuation of the kernel oscillations transversely to the path (Figure 1), so that the exact averaged kernel is accurately represented by a small number of oscillations that depends on the frequency width of the filter. The larger the frequency width, the more attenuated the kernel oscillations, while the central lobe remains essentially unchanged. We present the results obtained with 


\section{a) $2 D$ sensitivity zone}

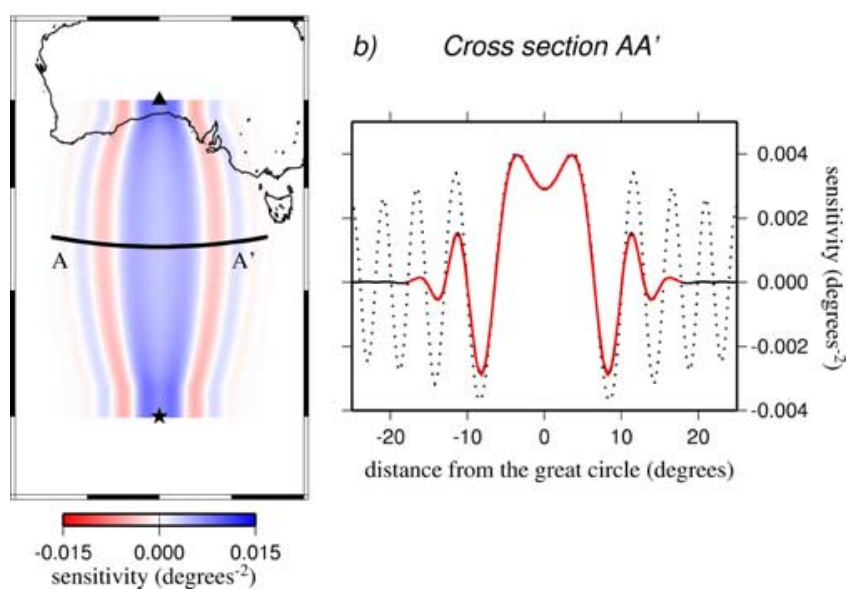

Figure 1. Scattering sensitivity kernel of the fundamental mode of a $160 \mathrm{~s}$ Rayleigh wave for a $30^{\circ}$ path a) $2 \mathrm{D}$ view, b) cross section at mid-distance: complete scattering kernel for a single frequency (dotted black line), with summation over a frequency-band (solid black line) and the truncated kernel eventually considered (red line).

a gaussian filter whose width at $-30 \mathrm{~dB}$ is equal to the central frequency. With this filter, a truncation at the fifth zero-crossing (Figure 1) is a very good approximation of the entire kernel. We therefore set $L(s)$ equal to the width of the fifth Fresnel zone, as defined by Spetzler et al. [2002].

\section{Synthetic Tests and Results}

[7] In the following tests, the synthetic data are fundamental mode Rayleigh wave phase velocities $c^{o b s}$ calculated at different periods using the finite-frequency theory summarized above (equation (2)) for realistic path distributions in a model with a plume-like perturbation (Figure 2). The path distributions shown in Figure 2 correspond to two different subsets of the path distribution used by Sieminski et al. [2003] in a regional surface wave tomography of Antarctica. The dense path coverage subset ensures a good coverage of the target, a plume-like perturbation, while the poor path coverage subset misses this perturbation entirely. In both cases, the path lengths range from 1200 to $9700 \mathrm{~km}$. a) Dense path coverage

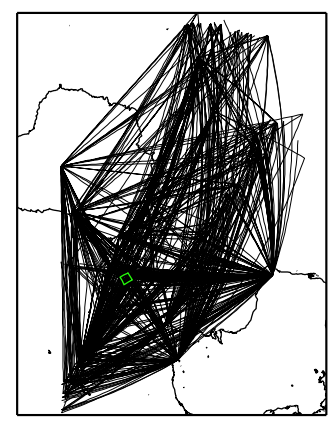

\section{b) Poor path coverage}

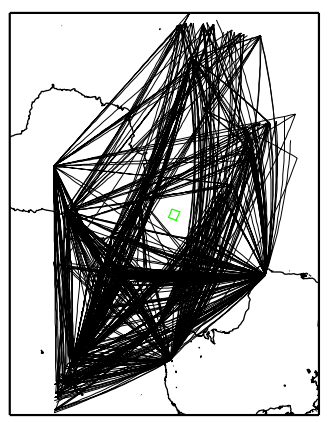

Figure 2. Path distributions of the test. The initial anomaly is a $-6 \% S$ wave velocity perturbation extending from Moho to $670 \mathrm{~km}$ depth with a width of $300 \mathrm{~km}$. Its initial position is marked by the green square.
[8] We inverted data both with great circle ray theory (equation (1)) and the finite-frequency theory (equation (2)), and repeated the tests twice, first assuming perfect data without noise and then adding random noise to the data. We found that adding noise reduces the differences between ray theory and finite-frequency results. Only the results from perfect data are presented here as they provide an upper bound on the relative effect of finite-frequency theory versus great circle ray theory.

[9] The great circle inversion is performed with the continuous regionalization algorithm of Montagner [1986] recently optimized by Debayle and Sambridge [2004] to process large data sets. An important parameter in our scheme is $L_{c}$, the correlation length between the model parameters, which controls the lateral smoothing of the inverted model. Only the points located within a 2D finite-size zone around each path contribute to the computation of the final model [Debayle and Sambridge, 2004]. The width of this "influence zone" is a function of $L_{c}$ (Figure 3a). Adapting $L_{c}$ to the wavelength of the data is thus a crude way to account for finite-size sensitivity zones, while still using ray theory framework. The 3D $S$ wave velocity model by Sieminski et al. [2003] was obtained by regionalizing a set of $1 \mathrm{D}$ depth-dependent path-average $S$ wave velocity models with $L_{c}=400 \mathrm{~km}$, corresponding to the average wavelength of the data set. In the following, the ray theory inversions are performed with this same $L_{c}$ value, which is comparable to the first Fresnel zone maximum width at $160 \mathrm{~s}$ for a $3300 \mathrm{~km}$ path, the average path length of the data set (Figure $3 \mathrm{~b}$ ). We have extended the Debayle and Sambridge's [2004] scheme to incorporate finitefrequency theory and use this modified scheme in our finite-frequency inversion. For the finite-frequency inversion, the overlap between the surfaces covered by the 2D sensitivity kernels centered around each path ensures a complete coverage of the area under study, rendering a priori lateral smoothing on the model unnecessary.

[10] Figure 4 shows the dense path coverage results for fundamental mode Rayleigh waves at $160 \mathrm{~s}$ using great

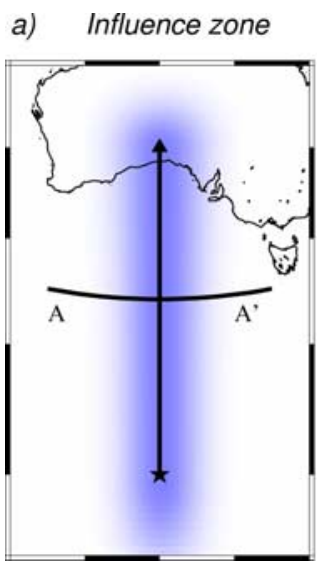

b) Cross section $A A^{\prime}$

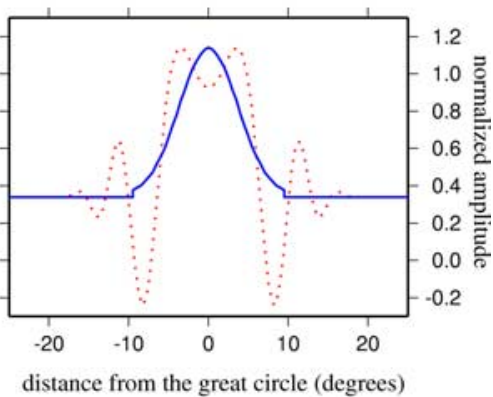

Figure 3. "Influence zone" related to $L_{c}$ for the same path as in Figure 1 with $L_{c}=400 \mathrm{~km}$ a) $2 \mathrm{D}$ view b) cross section at mid-distance. The shape of the "influence zone" transverse to the path is given by a gaussian (in blue), and the red dotted line is the truncated $160 \mathrm{~s}$ scattering sensitivity kernel of Figure 1 (amplitude normalized). 
a) GC Ray $160 \mathrm{~s}$

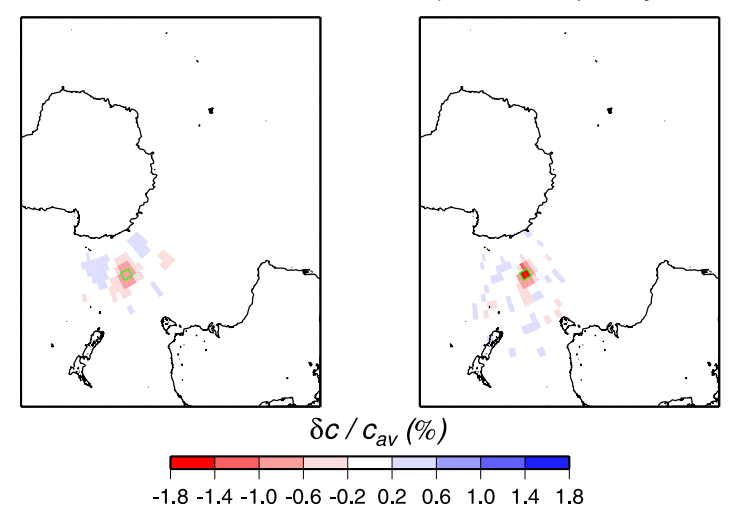

Figure 4. Models inverted in the case of a dense path coverage (Figure 2a) a) with great circle ray theory (GC Ray) and $L_{c}=400 \mathrm{~km} \mathrm{~b}$ ) with the finite-frequency theory (without further correlation between the model parameters) for the fundamental mode of a Rayleigh wave at $160 \mathrm{~s}$. The green square is the position of the input anomaly.

circle ray theory (Figure 4a) and finite-frequency theory (Figure 4b). Similar results are obtained for other periods. At $160 \mathrm{~s}$, despite the fact that the width of the input heterogeneity $(300 \mathrm{~km})$ is smaller than the wavelength $\left(\lambda_{160 s} \simeq 700 \mathrm{~km}\right)$, the plume-like anomaly is detected and correctly located with ray theory, suggesting that in this case the influence zone related to $L_{c}$ is an adequate description of the finite-size sensitivity zones. With finite-frequency theory (Figure 4b) the anomaly is in better focus and its amplitude is higher, however, the resulting model would not be interpreted any differently than the ray theory model. Moreover, the apparent superiority of the finite-frequency inversion in this case must be tempered for at least two reasons. First, in this noise-free case most of the difference between the two models is actually due to the smoothing effect of the ray theory parameter $L_{c}$. Indeed, applying the same a priori smoothing to the finite-frequency inversion leads to a very similar model to that obtained using ray theory. Second, tests carried out after adding noise to the perfect data showed that focusing and amplitude recovery in the finite-frequency inversion are significantly degraded.

[11] Let us now investigate what happens when the path coverage is degraded. Figure 5 shows the results obtained at $160 \mathrm{~s}$ and $100 \mathrm{~s}$ when the input plume-like anomaly is not crossed by any ray (Figure 2b) but still perturbs the synthetic data due to the finite-frequency effect. The amplitude of this perturbation depends on the width of the sensitivity zone and therefore on the period and path length. At $160 \mathrm{~s}$, ray theory with the $L_{c}$ influence zone (Figure 5a) retrieves a broad low velocity anomaly in the region of the input anomaly, while finite-frequency theory (Figure 5b) retrieves the anomaly much better. At $100 \mathrm{~s}$, the effect of the anomaly on the synthetic data is much smaller since, due to the poor coverage, the wave sensitivity to the anomaly is limited to a very marginal part of the kernel, where its amplitude is very small (Figure 1). At this period, while ray theory completely fails to retrieve the input anomaly (Figure 5c), a small low velocity perturbation is found at the correct location with finite-frequency theory (Figure $5 \mathrm{~d}$ ). However, the high performance of the finite-frequency inversion is dependent on the use of noiseless synthetic data computed with the same theory as used in the inversion, since adding noise to the data strongly weakens this result.

\section{Discussion}

[12] Several recent studies present comparisons of ray theory and finite-frequency tomography. For example, Montelli et al. [2004] obtain velocity perturbations up to $30-50 \%$ larger when finite-frequency effects are included in global $P$ and $P P$ travel time tomography, suggesting that wave front healing cannot be neglected when analyzing long period body waves. Discussing our results in terms of wave front healing is beyond the scope of this study. Spetzler et al. [2002] retrieve the large-scale structures $(>1000 \mathrm{~km})$ equally well by inverting Love wave phase shift data using ray theory or finite-frequency theory, whereas Ritzwoller et al. [2002], who approximate the central lobe of Spetzler et al.'s [2002] kernel by a boxcar transverse to the paths, find significant differences between finite-frequency and ray theory surface wave tomography at long periods and in regions covered only by long paths. As finite-frequency effects are indeed more important for long periods and long paths, and as our data set is limited to paths shorter than those for which Ritzwoller et al. [2002] observe the greatest differences, it is not surprising that we do not find as strong differences.

[13] Our tests point out the importance of the path coverage for obtaining high resolution images with ray theory. They suggest that heterogeneities smaller than a

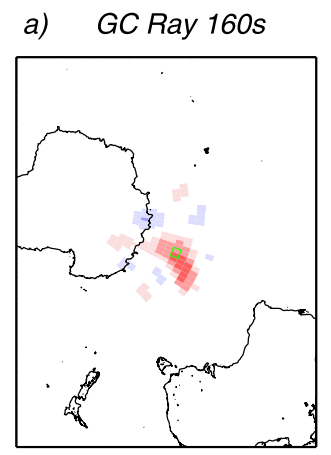

c) GC Ray 100s

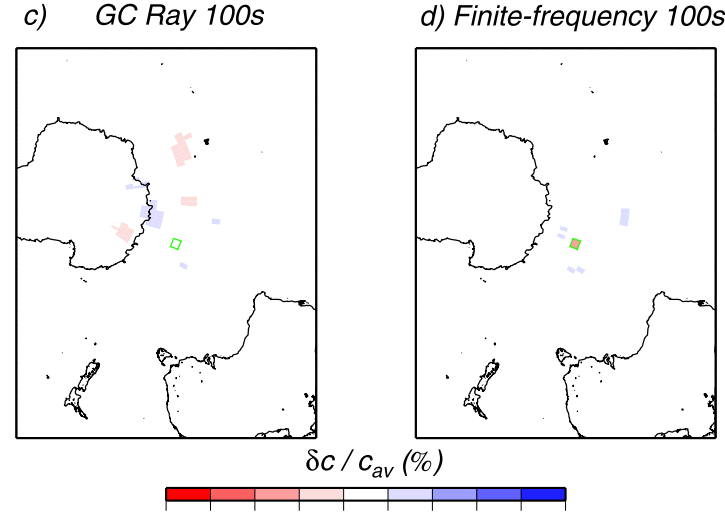

$\begin{array}{llllllllll}-0.5 & -0.4 & -0.3 & -0.2 & -0.1 & 0.1 & 0.2 & 0.3 & 0.4 & 0.5\end{array}$ b) Finite-frequency $160 \mathrm{~s}$

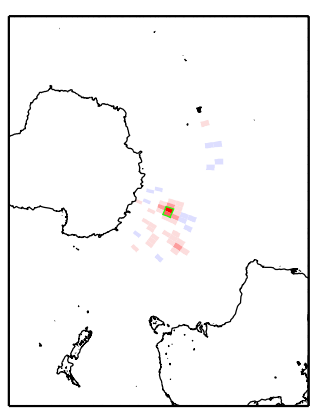

d) Finite-frequency 100 s
Figure 5. Same as Figure 4 but in a case of a very poor path coverage of the anomaly (Figure 2b) for the fundamental mode of a) and b) a $160 \mathrm{~s}$ Rayleigh wave and c) and d) a $100 \mathrm{~s}$ Rayleigh wave. 
wavelength or the first Fresnel zone width can be resolved with ray theory when combined with a physically-based regularization. This result is at first glance counterintuitive as ray theory is supposed to break down when the size of seismic heterogeneities is close to the wavelength or the Fresnel zone width. It is however in agreement with results from the recent migration experiment of Spetzler and Snieder [2004].

[14] Our regularization of the great circle ray theory inversion makes use of a "gaussian beam" centered around the great circle paths that defines an influence zone around the paths (Figure 3). The main difference with finitefrequency theory is that our influence zone is a gaussian function scaled by a fixed parameter $L_{c}$ whose width does not change along the path nor with the path length. Even in our test situation, where $50 \%$ of the paths are longer than the average $3300 \mathrm{~km}$ length, the single $L_{c}$ value of $400 \mathrm{~km}$ leads to results that are consistent with those of finite-frequency inversion, provided the path coverage is dense enough.

[15] Indeed, with this dense coverage, every part of the model is mainly sampled through the central lobe of the sensitivity kernel. The corresponding data perturbation is thus large enough to be conveniently described by approximate kernels such as a boxcar or a gaussian function. With the poor coverage (Figure 2b) however, the data perturbations due to the input anomaly are small, and obtained through the tiny sidelobes of the sensitivity kernel (Figure 1). Not surprisingly, only the use of the exact sensitivity kernels can correctly retrieve the anomaly (Figure 5). Note however that because of this weak sensitivity, any inaccuracy or noise in the data can prevent even the finite-frequency theory from retrieving the anomaly correctly.

[16] In the poor coverage case, slightly different images would be obtained with another choice for the frequency averaging of the kernels since the number of sidelobes depends on this width, but the qualitative conclusions would be the same. Conversely, the results for a dense coverage do not depend much on the frequency width, as the central lobe is not strongly affected by this parameter.

[17] Besides a poor path coverage, other effects can impair the anomaly retrieval with ray theory. For example, for surface wave tomographies that include a wide variety of path lengths, and where a single $L_{c}$ "influence zone" cannot mimic properly the variety of the $2 \mathrm{D}$ sensitivity zones, we expect more difficulties with ray theory. We have not tested the path bending effect that may be important especially for long paths [Ritzwoller et al., 2002].
[18] In conclusion, regional surface wave tomographies, which commonly meet the conditions of our experiments (i.e., good path coverage and short path lengths), can be safely conducted under the great circle ray theory assumption when combined with a proper inversion scheme. However, the case with which finite-frequency effects can be counterbalanced by using regularization parameters depends on the inversion technique. A regularization operator based on a priori physical assumptions, like in Tarantola and Valette [1982], is particularly well suited to the problem.

[19] Acknowledgment. This research is supported by program DyETI conducted by the French "Intitut National des Sciences de l'Univers" (INSU).

\section{References}

Debayle, E., and M. Sambridge (2004), Inversion of massive surface wave data sets: Model construction and resolution assessment, J. Geophys. Res., 109, B02316, doi:10.1029/2003JB002652.

Dziewonski, A., S. Bloch, and M. Landisman (1969), A technique for the analysis of transcient seismic signals, Bull. Seismol. Soc. Am., 59, 427444.

Montagner, J. P. (1986), Regional three-dimensional structures using longperiod surface waves, Ann. Geophys., 4, 283-294.

Montelli, R., G. Nolet, F. A. Dahlen, G. Masters, E. R. Engdahl, and S.-H. Hung (2004), Finite-frequency tomography reveals a variety of plumes in the mantle, Science, 303, 338-343.

Ritsema, J., H. van Heijst, and J. Woodhouse (2004), Global transition zone tomography, J. Geophys. Res., 109, B02302, doi:10.1029/ 2003JB002610

Ritzwoller, M. H., N. M. Shapiro, M. P. Barmin, and A. L. Levshin (2002), Global surface wave diffraction tomography, J. Geophys. Res., 107(B12), 2335, doi:10.1029/2002JB001777.

Sieminski, A., E. Debayle, and J.-J. Lévêque (2003), Seismic evidence for deep low-velocity anomalies in the transition zone beneath West Antarctica, Earth Planet. Sci. Lett., 216, 645-661.

Snieder, R. (2002), Scattering of surface waves, in Scattering and Inverse Scatterring in Pure and Applied Science, edited by R. Pike and P. Sabatier, pp. 562-577, Elsevier, New York.

Spetzler, J., and R. Snieder (2004), The Fresnel volume and transmitted waves, Geophysics, 69, 653-663.

Spetzler, J., J. Trampert, and R. Snieder (2002), The effect of scattering in surface wave tomography, Geophys. J. Int., 149, 755-767.

Tarantola, A., and B. Valette (1982), Generalised nonlinear inverse problems solved using the least square criterion, Rev. Geophys., 20, 219-232.

Trampert, J., and J. Woodhouse (1995), Global phase velocity maps of Love and Rayleigh waves between 40 and 150 seconds, Geophys. J. Int., 122, 675-690.

Yoshizawa, K., and B. Kennett (2002), Determination of the influence zone for surface wave paths, Geophys. J. Int., 149, 441-454.

E. Debayle, J.-J. Lévêque, and A. Sieminski, CNRS-UMR 7516-EOST, 5 rue René Descartes, F67084 Strasbourg Cedex, France. (anne.sieminski@ eost.u-strasbg.fr) 